\title{
Fabrication of PMMA X-ray Compound Refractive Lenses for the Pohang Light Source and Performance Tests
}

\author{
Guk Bae KIM and Sang Joon LEE* \\ Department of Mechanical Engineering, Pohang University of Science and Technology, Pohang 790-784 \\ Jin Pyung LEE \\ School of Environmental Science and Engineering, \\ Pohang University of Science and Technology, Pohang 790-784 \\ Jong Hyun Kim, Suk Sang Chang, Hwa Shik Youn and Tae Ju Sin \\ Pohang Accelerator Laboratory, Pohang University of Science and Technology, Pohang 790-784
}

(Received 15 May 2007)

\begin{abstract}
The majority of studies previously conducted on X-ray compound refractive lenses (CRLs) were related to synchrotron radiation (SR) facilities that had a relatively high photon energy level of several tens of $\mathrm{keV}$. However, the performance of CRLs at an SR source with the main photon flux at a photon energy of $7-9 \mathrm{keV}$, such as that of the Pohang Light Source (PLS), is still not very apparent. In this present study, to find the effective experimental conditions for CRL applications at the PLS, we focused the SR beam by using a CRL fabricated with poly methyl methacrylate (PMMA) under a photon energy of $8 \mathrm{keV}$. Various CRLs with different configurations were fabricated using the LIGA (LIthographie, Galvanoformung, Abformtechnik) process, and the focused beam size was measured at the PLS by using two different experimental methods. The size of the measured focused beam was $7.2 \mu \mathrm{m}$ at a focal length of $298 \mathrm{~mm}$. The CRLs developed in this study have a high potential and could eventually be used in real SR experiments at the PLS.
\end{abstract}

PACS numbers: 07.85.Qe, 41.50.+h

Keywords: X-ray focusing, Parabolic CRL, Kinoform, PMMA, LIGA process

\section{INTRODUCTION}

The X-ray compound refractive lens (CRL) [1] is an important tool for third-generation synchrotron radiation (SR) beams, together with other well-known methods for X-ray focusing such as Fresnel zone plates, Bragg-Fresnel optics, multilayer mirrors, planar waveguides, and capillary lenses. Various CRL materials have been tested under different X-ray energy levels. Planar parabolic lenses made of silicon have been tested at Xray energies of $8-25 \mathrm{keV}$ [2-5], and glassy carbon CRLs have been tested at $12.2 \mathrm{keV}$ and $25 \mathrm{keV}$ [6]. Several CRLs made of nickel [7], lithium [8], SU-8 polymer [9, 10] and PMMA [11-14] were also tested at various SR facilities.

The main factors governing CRL performance include lens material, fabrication method, lens configuration, and applied photon energy level. Most CRL studies were carried out under the condition of high photon energies of several tens of $\mathrm{keV}$ because CRLs at a low energy level

*E-mail: sjlee@postech.ac.kr; Fax: +82-54-279-3199 are known to be less effective than other X-ray focusing optics, such as zone plates. For the Pohang Light Source (PLS) (Pohang, Korea) which has a 2.5-GeV electron linear accelerator, the main photon energy flux appears in the range of $7-9 \mathrm{keV}$. Therefore, the photon energy level at the PLS is relatively low for employing CRL optics.

For the purpose of this study, compared with metal materials, a polymer is better in reducing the absorption of photons in the range of energy from $7-9 \mathrm{keV}$. In addition, lower energy X-rays can be focused within a shorter focal length. Some previous studies on CRL optics made of PMMA material were performed under an 8-keV photon energy level $[12,14]$. We carried out this study to improve the performance of PMMA CRLs and to optimize this X-ray focusing optics for use at the PLS facility. There was no trial to develop and employ this kind of refractive X-ray lens in the PLS. By employing the fabricated CRL, which is optimized for the PLS, we can enhance the resolution of X-ray micro-imaging and create new application researches.

To fabricate elaborate CRLs, we needed to select a pertinent lens material and optimize the CRL configura- 


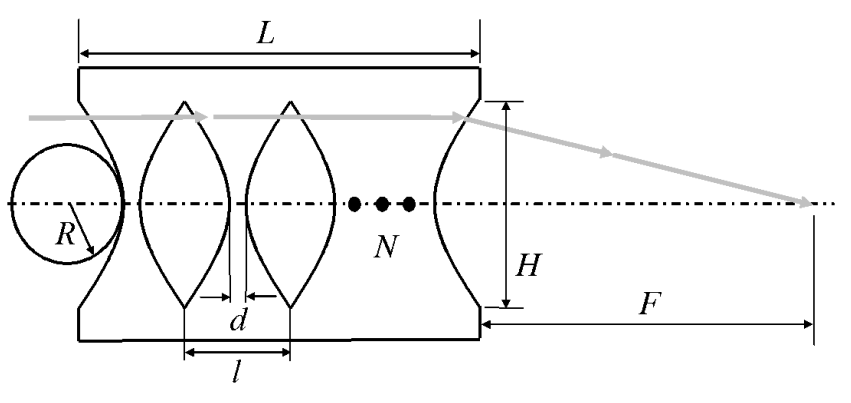

Fig. 1. Schematic diagram of a planar parabolic CRL. $F$ is the focal length of the CRL, $R$ is the parabola curvature radius, $N$ is the number of individual lens, $L$ is the total length of CRL, $H$ is the height of CRL, $l$ is the distance between adjacent lenses, and $d$ is the minimal distance between adjacent holes.

tion. In this study, PMMA was used as the CRL material, and CRLs of various configurations were fabricated using the LIGA fabrication method at the $9 \mathrm{C} 1$ beamline of the PLS. Two different measurement methods were employed for testing the performance of the developed CRLs under a photon energy of $8 \mathrm{keV}$ at the $1 \mathrm{~B} 2$ beamline of the PLS.

\section{BASIS OF X-RAY FOCUSING WITH CRL}

The refractive index $n$ of materials for X-rays is represented by $n=1-\delta+i \beta$. In general, the refractive index decrement $\delta$ denotes the degree of refraction in the vacuum condition, which has a positive value of $10^{-5}-10^{-7}$, depending on the constituent element and the density of the test material [15]. In addition, $\beta$ is related to the linear absorption coefficient $\mu$ and to the photon wavelength $\lambda$ as

$$
\beta=\frac{\lambda \mu}{4 \pi} .
$$

The refractive index decrement $\delta$ depends on the photon energy. Therefore, we need to use lenses of a concave shape to focus X-rays because $n$ is less than 1 . The refractive index of a PMMA material for X-rays is very low. Therefore, many lenses should be compounded or integrated, as shown in Figure 1, to reduce focal the length. The focal length $F$ can be calculated using the formula $F=R / 2 N \delta[16]$.

In general, the geometry of a circular-shaped lens causes spherical aberration. On the other hand, a parabolic-shaped lens can enhance the focusing performance, and its length is much smaller than that of a corresponding circular lens [17]. Therefore, the geometry of all CRLs tested in this study was designed to have a parabolic shape. The parabola's curvature radius $R$ can be determined using the formula $l-d=(H / 2)^{2} / R$ $[18]$. (a)
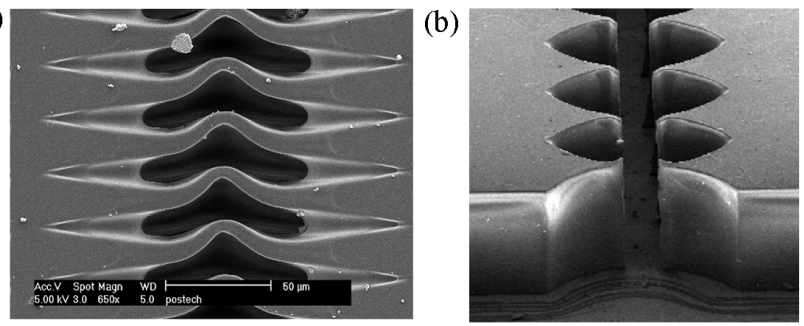

Fig. 2. Micrographs of planar CRLs fabricated under unsuitable conditions.

The image in the focal plane of a CRL represents the scalar sum of light intensities of all points of the beam source. Therefore, the size of the focused beam on the image can be evaluated using the laws of geometrical optics as follows:

$$
S_{F}=\frac{F}{S} \times S_{S R}
$$

Here, $S_{F}$ is the beam size focused by the CRL located at the focal plane while $S$ is the distance from the source to the CRL, and $S_{S R}$ is the size of the SR source.

\section{DESIGN AND FABRICATION OF CRL}

In this study, we fabricated two different groups of CRLs planar parabolic CRLs and kinoform CRLs. All the fabricated CRLs were designed to have a fixed focal length of $296 \mathrm{~mm}$ in order to easily compare their performances. The focal length $F$ can be adjusted by varying the number of individual lenses $N$ or the radius of the parabola curvature $R$. With a fixed focal length $F$ and CRL height $H$, a large curvature $R$ denotes a decrease in the X-ray absorption area. However, an excessively large $R$ requires an excessive number of lenses $N$ and excessive wall thickness $d$. Moreover, a CRL with a large $R$ and a fixed $H$ brings about sharp edges in the individual holes of the CRL. In the LIGA fabrication process, a sharp edge can cause severe problems of underdevelopment of the lens material at both edges of each hole, as shown in Figure 2(a). The wall thickness $d$ between adjacent holes should also be considered as one of the important parameters in the LIGA fabrication process. In general, a thinner $d$ is better in the viewpoint of X-ray absorption. However, an excessively thin $d$ can cause deformation or over-development of the walls, as shown in Figure 2(b).

To find the optimum values of the governing parameters $R$ and $d$ for the LIGA fabrication process, we designed CRLs of various configurations with different values of $R$ and $d$. The radius $R$ of planar parabolic CRLs was varied from 20 to $400 \mu \mathrm{m}$, and the corresponding number of lenses $N$ ranged from 8 to 160 at a fixed focal length of $296 \mathrm{~mm}$. The minimal distance $d$ between the adjacent holes was varied from 1 to $20 \mu \mathrm{m}$. The depth and the height $H$ of the planar CRLs were fixed at 500 


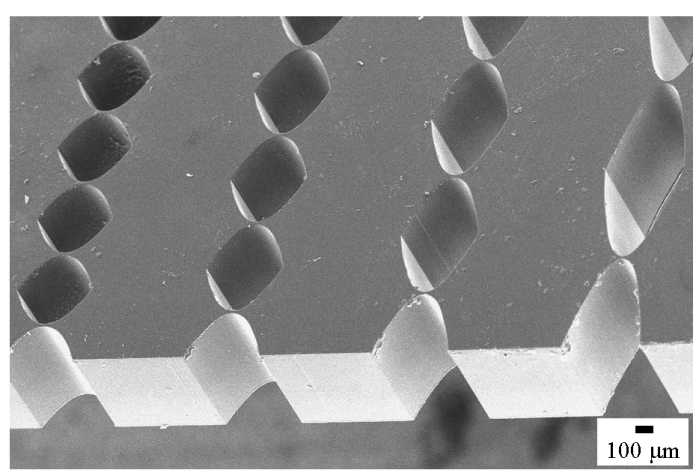

Fig. 3. SEM micrograph of a fragment of the planar parabolic CRLs fabricated in this study. From the left side, the radii of curvature of the parabola, $R$, are 100, 80, 60 and $40 \mu \mathrm{m}$, respectively.

$\mu \mathrm{m}$ and $400 \mu \mathrm{m}$, respectively. From a simple inspection of the fabricated CRLs, the optimum conditions for which the planar CRL could be well fabricated and were sufficiently strong were found to be $R=40-100 \mu \mathrm{m}$ and $d=17 \mu \mathrm{m}$. Figure 3 shows a typical SEM micrograph of a fragment of the planar parabolic CRLs fabricated in this study.

In the case of kinoform CRLs on a parabola, the key parameters for the LIGA fabrication process were the radius of parabola curvature $R$ and the number of kinoform segments $S$. The radius $R$ of the kinoform CRLs was varied from 10 to $100 \mu \mathrm{m}$, and the corresponding number of lenses $N$ ranged from 8 to 80 at a fixed focal length of $296 \mathrm{~mm}$. The depth and the height $H$ of all the kinoform fabricated CRLs were fixed at $500 \mu \mathrm{m}$ and $400 \mu \mathrm{m}$, respectively. The numbers of segments $S$ tested in this study were 7, 9 and 15 . Theoretically, the outer segment of the kinoform CRLs should have a sharper edge. However, it is difficult to fabricate such an extremely sharp edge when using the LIGA process. Moreover, as the radius $R$ is decreased, the edge becomes sharper. Therefore, a kinoform CRL with a large $R$ would be better than a kinoform CRL with a small $R$. As the number of segments $S$ increases, the X-ray absorption area is reduced. However, an excessively large value of $S$ would increase the number of sharp edges. Upon inspection of the fabricated CRLs, the conditions of $R=100 \mu \mathrm{m}$ and $S=7$ were found to be proper for fabricating kinoformshaped CRLs.

The LIGA fabrication process of CRLs is based on Xray lithography which facilitates accurate replication of a pattern from a mask (thin membrane) into a substrate by means of chemical changes induced by SR exposure. The mask with a 2-D absorber pattern acts as a slide, and SR irradiation allows precise and deep shadow printing on the resist due to the good straightness and high intensity of the SR beam.

A schematic diagram of the LIGA process for fabricating PMMA CRLs is shown in Figure 4. In the X-ray mask fabrication, a $\mathrm{Cr} / \mathrm{Au}$ seed layer was deposited on

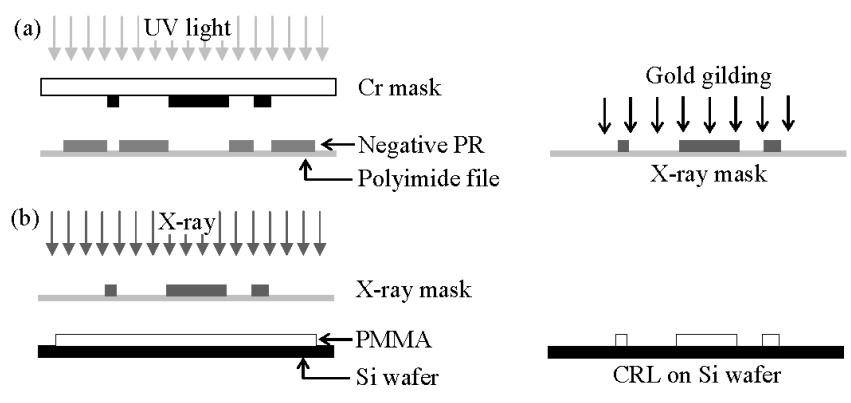

Fig. 4. Schematic diagram of the LIGA process for fabricating PMMA CRLs.

the membrane film by means of e-beam evaporation. The membrane film should be made of a material that minimizes X-ray loss through absorption. It must also be mechanically robust to ensure high fabrication yields. In general, silicon wafers, $\mathrm{SiO}_{2}$, or $\mathrm{Si}_{3} \mathrm{~N}_{4}$ membranes satisfy these requirements. However, because it is more desirable to reduce the gap between the chrome mask and the membrane during ultraviolet (UV) exposure and to minimize the lateral dimension errors, we used a thin polyimide film as a substrate. The negative photoresist (PR) was uniformly spin-coated on the $\mathrm{Cr} / \mathrm{Au}$ deposited substrate. The PR-coated substrate was then soft baked on a hot plate to minimize and cure the residual stress accumulated on the resist during the spin coating process. The resist was patterned with a chrome mask under UV exposure at wavelengths of $365 \mathrm{~nm}$ and $405 \mathrm{~nm}$. The exposed pattern was baked again and then developed. The residual $\mathrm{PR}$ and developer were removed by using an $\mathrm{O}_{2}$ plasma to keep the resist surface clean for the subsequent electroplating process. Gold was electro-deposited in the resist cavity to make an X-ray mask with an average thickness of approximately $20 \mu \mathrm{m}$. After removing the PR, an inverse lens-shaped gold absorber having the pattern of the X-ray mask was obtained, as shown in Figure 4(a).

In the fabrication of microstructures using X-ray lithography, the PMMA material has been commonly used. In this study, the PMMA material was adhered to a silicon wafer in the form of sheet casting to fabricate CRLs. It was then adhered to adhesion promoters such as S1805 PR. Liquid PMMA and MMA were used for bonding the two layers. The X-ray exposure was carried out at the $9 \mathrm{C} 1 \mathrm{X}$-ray nano/micromachining (XNMM) beamline at the PLS. Three-dimensional microstructures of CRLs were obtained by projecting the 2-D absorber pattern on the mask onto the PMMA resist, leaving a latent image in the exposed part, which was then developed after irradiation. Related with the developing process, the dose rate was controlled to within $4 \mathrm{~kJ} / \mathrm{cm}^{3}$ at the bottom. After the chemical development of PMMA, a final CRL-shaped PMMA structure was obtained, as shown in Figure 4(b). 




Fig. 5. Two experimental methods for measuring the size of the focused beam.
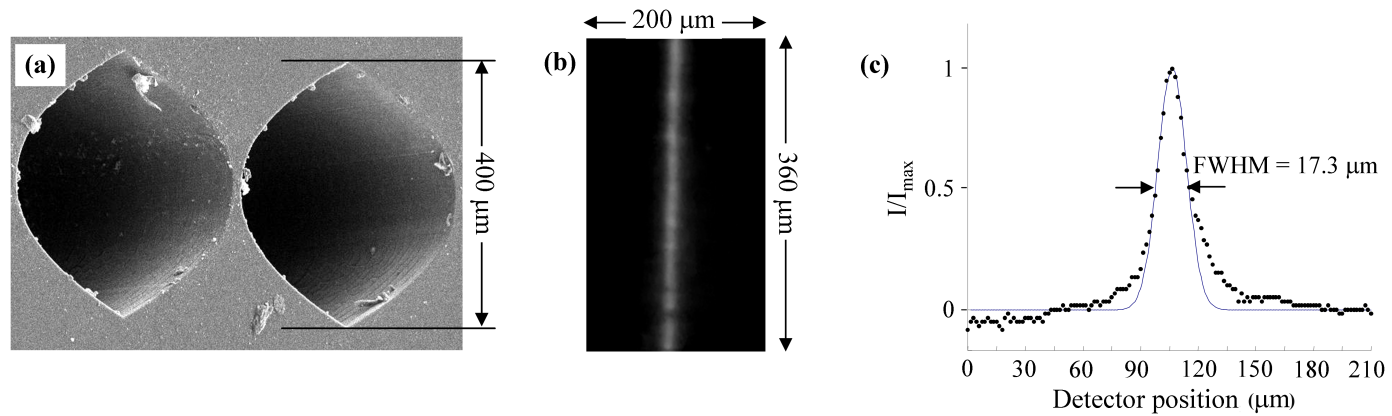

Fig. 6. Micrograph and performance of the N-CRL tested with the CCD imaging setup at a photon energy of $8 \mathrm{keV}$ : (a) micrograph of CRL tested, (b) line image of the focused beam, and (c) light intensity profile extracted from the line image.

\section{PERFORMANCE TESTS}

In order to measure the beam size focused by the fabricated CRLs, we established two different kinds of experimental setups at the bending magnet beamline 1B2 of the PLS. The schematic diagram of the two experimental setups is shown in Figure 5. The horizontal and the vertical cross-sectional sizes of the SR source were 160 $\mu \mathrm{m}$ and $60 \mu \mathrm{m}$, respectively. The source-to-CRL distance was $24 \mathrm{~m}$. The photon energy of the X-ray source measured with a Si 4-crystal channel-cut monochromator was $8 \mathrm{keV}$. At first, a line image focused by the employed CRL was obtained on the scintillation crystal placed at the focal plane. The focused line image on the scintillation crystal was captured using a CCD (charge coupled device) camera, in front of which a $10 \times$ objective lens was attached. The spatial resolution of the imaging recording system was about $2-3 \mu \mathrm{m}$, and the exposure time for capturing one image was about $33 \mathrm{~ms}$. In truth, this imaging method using a scintillation crystal is not very effective for measuring the absolute value of the focused beam size. However, it is useful in comparing the focusing performance of CRLs because the CCD detector can capture a number of focused line images easily and quickly. This CCD imaging method was mainly used for finding the best CRL from among the many planar and kinoform CRLs tested in this study.

Second, the focal distance and the focused beam size were precisely measured using a knife-edge scan method [19]. A tungsten wire, $30 \mu \mathrm{m}$ in diameter, was traversed horizontally across the focused beam, and the derivative of the knife-edge scan signal was used. An ionization chamber, 95-mm long and located downstream of the tungsten wire, was used to measure the beam intensity. The focused beam size is usually represented by the full width at half maximum (FWHM) of the derivative function of the light intensity profile in the knife-edge scan signal. The focused beam size was determined as the minimum FWHM of the output beam.

From the visual inspection and the performance tests using the CCD imaging method, the effect of the governing parameters on the finished product and the performance of the fabricated CRLs were investigated. The main parameters of the two CRL groups tested in this study are summarized in Table 1 . Here, the normal CRL (denoted hereafter as N-CRL) represents the group of planar parabolic CRLs, and the kinoform CRL (denoted hereafter as K-CRL) indicates the group of kinoform CRLs on parabolas. Because the N-CR has double-sided lenses, the number of the N-CRL is double that of the K-CRL.

The micrographs of the tested N-CRL and K-CRL and their performance results are shown in Figures 6 and 7, respectively. As Figures 6(a) and 7(a) show, the distance between two adjacent lenses of the N-CRL is longer than that of the K-CRL. This means that the effective absorbing area of the lens material for the K-CRL is much smaller than that of the N-CRL. The total length $L$ of the $\mathrm{K}$-CRL is $9.6 \mathrm{~mm}, \sim 43 \%$ shorter than that of the $\mathrm{N}$-CRL $(16.8 \mathrm{~mm})$. Using the CCD imaging setup, we 
(a)

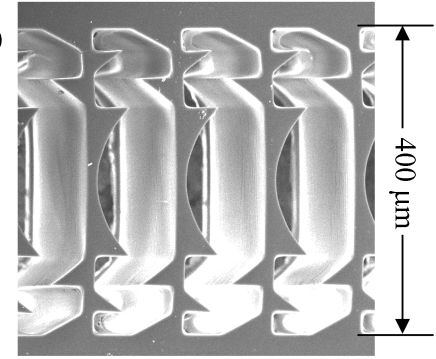

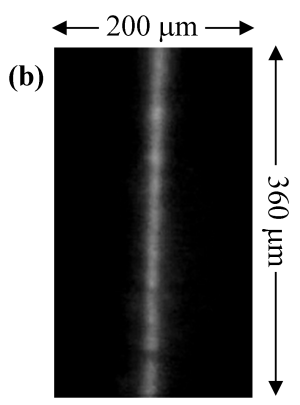

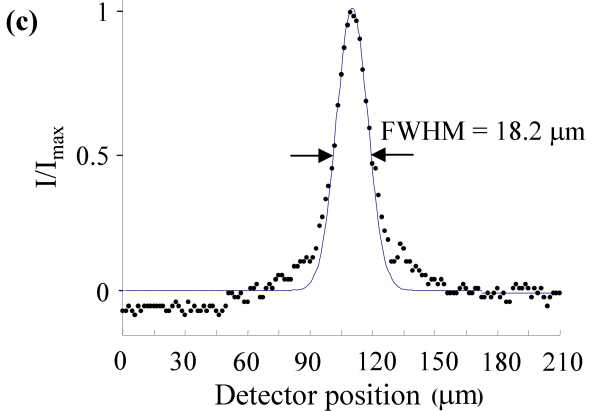

Fig. 7. Micrograph and performance of the K-CRL tested with the CCD imaging setup at a photon energy of 8 keV: (a) micrograph of CRL tested, (b) line image of the focused beam, and (c) light intensity profile extracted from the line image.

Table 1. Parameters of the CRLs tested.

\begin{tabular}{lccccc}
\hline \hline Name of lens & $\begin{array}{c}\text { Number of } \\
\text { lens, } N\end{array}$ & $\begin{array}{c}\text { Radius of } \\
\text { curvature, } R(\mu \mathrm{m})\end{array}$ & $\begin{array}{c}\text { Distance between } \\
\text { holes, } d(\mu \mathrm{m})\end{array}$ & $\begin{array}{c}\text { Number of } \\
\text { segments, } S\end{array}$ & $\begin{array}{r}\text { Calculated focal } \\
\text { length, } F(\mathrm{~mm})\end{array}$ \\
\hline Normal CRL (N-CRL) & 40 & 100 & 17 & - & 296 \\
Kinoform CRL (K-CRL) & 80 & 100 & 17 & 7 & 296 \\
\hline \hline
\end{tabular}

captured line images of the focused beam at the estimated focal plane of $F=296 \mathrm{~mm}$. Figures $6(\mathrm{~b})$ and 7(b) show the focused line images. The light intensity profiles were extracted from the line images, as shown in Figures $6(\mathrm{c})$ and $7(\mathrm{c})$. The FWHM value of the focused beam for the N-CRL and the K-CRL lenses are $17.3 \mu \mathrm{m}$ and $18.2 \mu \mathrm{m}$, respectively.

It is worthwhile to note that the FWHM of the K-CRL is a little larger than that of the N-CRL, even though the $\mathrm{K}-\mathrm{CRL}$ is known to have a better focusing performance than the N-CRL. This may be attributed to the facts that the sharp edges of the K-CRL could not be welldeveloped during the fabrication process and that the focusing X-ray beam could expand slightly at the gap between adjacent segments as the X-rays pass through many individual lenses. From this study, we can figure out that it is really difficult to make a proper kinoformtype CRL that has good focusing efficiency. This is mainly because many sharp-edged configurations are required to be able to form a precise kinoform shape. From a simple comparison using the CCD imaging setup, the N-CRL seems to show a good performance among the CRLs tested in this study.

On the other hand, we established that the theoretical value at the focal plane, $S_{F}$, was $1.97 \mu \mathrm{m}$ when we estimated the beam size of the N-CRL by using Eq. (2) with the conditions of $F=296 \mathrm{~mm}, S=24 \mathrm{~m}$, and $S_{S R}$ $=160 \mu \mathrm{m}$ in the horizontal direction. Compared to the theoretical beam size of $S_{F}=1.97 \mu \mathrm{m}$, the experimental result for the N-CRL $(17.3 \mathrm{~m})$ measured using the CCD imaging method was very large. One of the main reasons for this large difference is the fact that the captured line image was blurred inside the scintillation crystal. In addition, the line image was measured at the theoreti- cally estimated focal length instead of the actual focal length location. As we previously mentioned, because the CCD imaging method is only employed for finding pertinent parameters of CRLs with a simple performance comparison, the line image data cannot verify the real performance of the CRLs fabricated in this study.

To accurately measure the size and the focal length of the beam focused by the N-CRL, we employed the tungsten wire method. The beam size was measured at seven positions ranging from $283 \mathrm{~mm}$ to $313 \mathrm{~mm}$ from the center location of the CRL. Figure 8(a) shows the variation of the beam size focused by the N-CRL as a function of the distance from the CRL center. The parabolic fitting curve shows that the focal length was located at a position of about $298 \mathrm{~mm}$ and that the FWHM at the focal length was estimated to be about $7.2 \mu \mathrm{m}$. Figure 8(b) shows the light intensity profile of the focused beam measured by using an ionization chamber at a distance of $295 \mathrm{~mm}$, at which the FWHM has the smallest value of $7.3 \mu \mathrm{m}$ among the experimental results. This value is a remarkably improved result, compared with the previous other results of $16 \mu \mathrm{m}$ [12] and $120 \mu \mathrm{m}$ [14]. The measured focal length of $298 \mathrm{~mm}$ is sufficiently well matched with the calculated focal length of $296 \mathrm{~mm}$. However, the size of the focused beam $(7.2 \mu \mathrm{m})$ is still larger than the theoretically predicted value $\left(S_{F}=1.97 \mu \mathrm{m}\right)$. This may be attributed to the broadening of the focused beam due to imperfections in the monochromator crystals, smallangle scattering from the heterogeneities of the PMMA material, surface roughness on the parabolic lens shape, and so on.

We also theoretically estimated the depth of field $d_{1}$ to check the longitudinal resolution. From Eq. (1), the linear absorption coefficient $\mu$ of the PMMA material is 
(a)



(b)

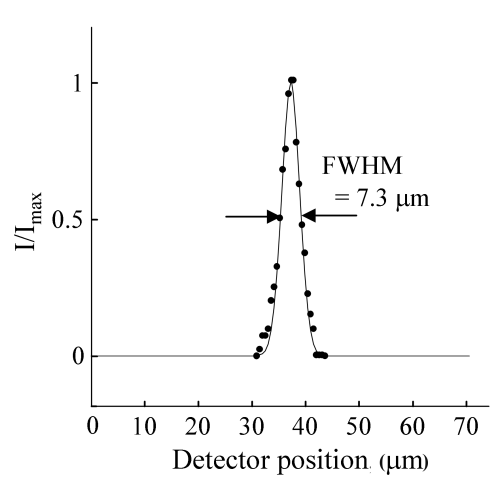

Fig. 8. Size of the focused beam measured by using a tungsten wire setup at a photon energy of $8 \mathrm{keV}$ : (a) variation of the beam size focused by N-CRL and (b) profile of the focused beam at a distance of $295 \mathrm{~mm}$.

$7.422 \mathrm{~cm}^{-1}$ at the $1.55-\AA$ wavelength of $8 \mathrm{keV}$. In the following equations, the effective aperture $D_{\text {eff }}$ is 16.4 $\mu \mathrm{m}$ at $R=100 \mu \mathrm{m}$ and $N=40$, the CRL-to-image distance $L_{2}$ is $299.7 \mathrm{~mm}$, and the numerical aperture $N A$ is $2.74 \times 10^{-4}$.

$$
D_{e f f}=2 \sqrt{2 R / \mu N}, L_{2}=\frac{f \times L_{1}}{L_{1}-f}, N A=\frac{D_{e f f}}{2 L_{2}} .
$$

From the following Eq. (3) [20], the depth of field $d_{1}$ of the tested N-CRL is about $2.07 \mathrm{~mm}$ and the estimated depth of field seems to fit suitably the fitting curve in Figure 9(a):

$$
d_{1}=0.64 \frac{\lambda}{(N A)^{2}} \text {. }
$$

\section{CONCLUSION}

Two different types of CRLs, namely, the normal parabolic CRL (N-CRL) and the parabolic-shaped kinoform CRL (K-CRL), were fabricated using the LIGA process at the third-generation SR of the PLS. Their performance was tested by employing two different measurement methods. Even though the PLS irradiates at a relatively low photon energy level for applying CRL optics, we tried to focus the PLS X-rays at a photon energy of $8 \mathrm{keV}$. The fabricated CRL optics made of PMMA material were well fabricated using the LIGA fabrication process. Moreover, they seemed to be effective at the PLS with regard to their low absorption rate and relatively high refractive index at the low photon energy level of X-rays. From a simple comparison of the fabrication results, the optimum conditions for which the CRL can be well fabricated with a sufficiently strong structure are $R=100 \mu \mathrm{m}$ and $d=17 \mu \mathrm{m}$ for the N-CRL and $S=7$ for the K-CRL. From the performance test using a CCD imaging setup, the N-CRL was found to show a better performance than the K-CRL. Using the tungsten wire method, we measured the focused beam size of the NCRL as $7.2 \mu \mathrm{m}$ at a focal length of $298 \mathrm{~mm}$. Therefore, the CRLs fabricated in this study can be used for microfluorescence and micro-EXAFS experiments at the PLS. They may also find applications in the LIGA process to fabricate microstructures of sub-micron size.

\section{ACKNOWLEDGMENTS}

Experiments at the $1 \mathrm{~B} 2$ and the $9 \mathrm{C} 1$ beamline of the PLS were supported in part by the Korea government of Ministry of Science and Technology (MOST) and Pohang University of Science and Technology. The present study was financially supported by the Korea Science and Engineering Foundation (KOSEF) grant funded by MOST (No. R01-2004-000-10500-0 of the Basic Research Program and No. R15-2004-033-06002-0 from Systems Bio-Dynamics Research Center).

\section{REFERENCES}

[1] T. Tomie, X-ray lens, United States Patent No. 5594773, 1997.

[2] V. Aristov, M. Grigoriev, S. Kuznetsov, L. Shabelnikov and V. Yunkin, Appl. Phys. Lett. 77, 4058 (2000).

[3] I. Snigireva, A. Snigireva, C. Raua, T. Weitkampa, V. Aristov et al., Nucl. Instr. and Meth. A 467, 982 (2001).

[4] V. V. Aristov, M. V. Grigoriev, S. M. Kuznetsov, L. G. Shabelnikov, V. A. Yunkin, M. Hoffmann and E. Voges, Opt. Comm. 177, 33 (2000).

[5] C. G. Schroer, M. Kuhlmann, U. T. Hunger, T. F. Gunzler and O. Kurapova et al., Appl. Phys. Lett. 82, 1485 (2003).

[6] A. N. Artemiev, A. Snigirev, V. Kohn, I. Snigireva and N. Artemiev et al., Nucl. Instr. Meth. A 543, 322 (2005).

[7] V. Nazmov, E. Reznikova, A. Snigirev, I. Snigireva and M. DiMichiel et al., Micosyst. Technol. 11, 292 (2005). 
[8] N. R. Pereira, E. M. Dufresne, R. Clarke and D. A. Arms, Rev. Sci. Instrum. 75, 37 (2004).

[9] V. Nazmov, E. Reznikova, J. Mohr, A. Snigirev and I. Snigireva et al., Microsyst. Technol. 10, 716 (2004).

[10] V. A. Chernov, K. E. Kuper, A. A. Legkodymov, V. V. Lyakh and V. F. Pindyurin et al., Nucl. Instr. Meth. A 543, 326 (2005).

[11] R. K. Kupka, F. Bouamrane, M. Roulliay and S. Megtret, SPIE. 3680, 508 (1999).

[12] M. A. Piestrup, J. T. Cremer, H. R. Beguiristain, C. K. Gary and R. H. Pantell, Rev. Sci. Instrum. 71, 4375 (2000).

[13] V. Nazmov, L. Shabel'nikov, F. J. Pantenburg, J. Mohr, E. Reznikova and A. Snigirev et al., Nucl. Instr. Meth. B 217, 409 (2004).
[14] Y. Ohishi, A. Q. R. Baron, M. Ishii, T. Ishikawa and O. Shimomura, Nucl. Instr. Meth. A 467, 962 (2001).

[15] S. Suehiro, H. Miyaji and H. Hayashi, Nature 352, 385 (1991).

[16] A. Snigirev, V. Kohn, I. Snigireva and B. Lengeler, Nature 384, 49 (1996).

[17] G. I. Kweon and C. H. Kim, J. Korean Phys. Soc. 51, 93 (2007).

[18] C. G. Schroer and B. Lengeler, Phys. Rev. Lett. 94, 054802 (2005).

[19] F. A. Hofmann, C. A. Freinberg-Trufas, S. M. Owens, S. D. Padiyar and C. A. MacDonald, Nucl. Instr. Meth. B 133, 145(1997).

[20] B. Lengeler, C. G. Schroer, B. Benner and T. F. Gunzler et al., Nucl. Instr. Meth. A 467, 944 (2001). 